\title{
Processes of Obtaining Building Materials and Their Study Through the Theory of Scattered Systems
}

\author{
L.C. Moreno-Chimely, Y.G. Aranda-Jiménez, J.F. Pérez-Sánchez*, I. Anchondo-Pérez, E.J. \\ Suárez-Domínguez*, M.A. Varela-Tovar, J.G. Lorenzo-Palomera. \\ ${ }^{1}$ Faculty of Architecture, Design and Urban planning. The Autonomous University of Tamaulipas. Circuito \\ Interior S/N Centro Universitario Sur. Tampico, Tamaulipas. México. \\ *Corresponding author: josue.perez@uat.edu.mx
}

\begin{abstract}
Building materials must meet several requirements so that they can be used in a given application. These requirements are determined from the measurement of physical properties, depending on the nature of the components. This paper presents a set of mathematical processes and analyses to be carried out for new materials development according to the theory of dispersed or polydispersive systems, including a set of proposed steps to characterize such materials as the criteria for the study. Studies are considered at the microscopic level from the interaction of the elements and the macroscopic level from properties for emulsion cases, including viscosity.
\end{abstract}

Key words: construction materials; cementitious dispersed system; theoretical materials analysis.

\section{INTRODUCTION}

The evaluation of materials is currently essential because their characteristics allow them to decide their use in various aspects of human life [1, 2]. The processes for this are diverse, and this work aims to present some of the strategies and evaluations mainly of the theoretical-experimental field, which they obey.

Physical properties that are measured to characterize the quality of building materials include:

Microscopic scale structure: This property is closely related to the molecular properties of the components that make up the material [3], and can generally be classified as crystalline structures, where molecules are spatially organized, and vitreous structures characterized by molecular disorder. Materials typically adopt a hybrid system, where both types of structures are appreciated [4].

Macroscopic structure: According to their appearance, the following types of designs can be distinguished [5-7]:

$\checkmark$ Compact: when they have a homogeneous mass. (Marbles)

$\checkmark$ Porous or cellular: they have a heterogeneous mass with pores, cavities, and organic remains. (Pómez
Stone)

$\checkmark$ Granular: in the form of agglomerated grains. (Sandstones)

$\checkmark$ Stratified: they have bands according to sedimentation layers (Clays)

$\checkmark$ Laminar: formed by thin sheets of easy exfoliation. (Slates)

$\checkmark$ Classic: formed by fragments agglomerated by natural cement (Conglomerates)

$\checkmark$ Fibrous: forming parallel or radial fibers, being needles and capillary (fine threads). (gypsum)

$\checkmark$ Porphydic: large granules stand out on a paste of minerals that are difficult to tell. (Porphydic)

$\checkmark$ Granitoids: present minerals in attached grains of similar sizes. (Granites)

General definitions to consider include:

Porosity: Refers to the volume fraction of the material that is hollow. Porosity is due to the manufacture of the building materials forming a suspension subjected to a drying process so that the evaporated water leaves a space occupied by the air.

Volume: The apparent volume of a material is the sum of the volume occupied by the solid and the volume occupied by the closed pores (with no communication with the outside). The total volume is the sum of the apparent volume and the volume occupied by the open pores, which correspond with the outside and manifest themselves on the material's surface.

Density: Se defines as mass per unit volume and is determined experimentally. Depending on the volume considered, there are three different types of density: the actual density, which only considers the solid volume, the apparent density that is determined by the apparent volume, and the overall density, which is calculated with the total volume.

Porosity is one of the most important properties determining the quality of given building material since it influences its mechanical properties, chemical attack resistance, and thermal conductivity associated with the houses' comfort.

Porosity decreases fracture resistance, as pores reduce the area through which the load is applied while acting as tension 
concentrators, amplifying it. Se has shown that the breakage module decreases exponentially with the pore volume fraction $(P)$ according to to the Ryshkevich formula [8-10]:

$$
\sigma_{m f}=\sigma_{0} \exp (-n P)
$$

where $\sigma_{0}$ represents the break module of the non-porous material, and $n$ is a constant that is determined experimentally.

The elasticity module also decreases with ratio porosity [11-13]:

$$
E=E_{0}\left(1-1.9 P-0.9 P^{2}\right)
$$

The building materials are solids formed by a mixture of different components, which must have a homogeneous spatial composition [14,15]. Because their manufacture is based on mixing solid elements with water, pouring the mixture into a mold, and proceeding to the drying process, these can be considered scattered soli, where air occupies the space occupied by the water during drying process is completed. For this reason, porosity (related to the strength and elasticity properties) depends on the composition and the way the material is manufactured.

Since the manufacturing process begins by forming a suspension [16] from which a solid sun is created, it is interesting to understand the general properties of the systems of the product, since this allows to understand better the chemical-physical processes that occur and their relationship to the quality of the final product.

\subsection{General Characteristics of Scattered Systems}

The properties exhibited by systems formed by different components depend on the chemical-physical properties of each of the elements and the interactions established between them at the molecular level[17-19]. When components have similar molecular structures and are in a liquid state, they can interact with each other by mixing them, meaning that molecules surround molecules of components in lower proportion (solute) in a more significant proportion (solvent). If the components have molecular structures with markedly different moments, they do not interact, so the components are separated into immiscible phases. Due to the effects of surface tension, the area of contact between phases or interfacial areas tends to take a minimum value, implying a total separation between stages that can be distinguished at the macroscopic level, meaning that the system's composition depends on the spatial position.

Scattered systems are composed of two or more phases immiscible to each other [20] distributed in such a way that one of the phases (dispersed phase) is contained in the other (dispersion medium), forming small features so that the composition is independent of the spatial position. These can be classified according to the following criteria:
The number of phases: can be systems consisting of only two phases or several phases (multiphase).

Scattered particle size: Particles with a size greater than $0.003 \mathrm{~cm}$ are classified as dispersed systems, while when the particles are smaller, they are classified as colloidal systems. Colloidal systems are characterized by behaving as macroscopic monophase systems while exhibiting a set of characteristic properties that distinguish them from single-phase systems.

Size distribution of dispersed particles: mono-dispersed (all equal-sized particles) or polydispersed (particles have different sizes).

Aggregation status: these systems are classified as shown in Table 1 according to the authors [21-23].

Table 1. Classification of dispersed systems according to aggregation status [21-23]

\begin{tabular}{|c|c|c|c|}
\hline Classification & $\begin{array}{c}\text { Scattering } \\
\text { Media }\end{array}$ & $\begin{array}{c}\text { Dispersed } \\
\text { phase }\end{array}$ & Example \\
\hline $\begin{array}{c}\text { Liquid } \\
\text { Aerosol }\end{array}$ & Gas & Liquid & Mist \\
\hline Solid Aerosol & Gas & Solyd & Smoke \\
\hline Foams & Liquid & Gas & Foams \\
\hline Emulsions & Liquid & Liquid & Mayoinnaise \\
\hline Suspension & Liquid & Solid & Paints \\
\hline Sol-Solid & Solid & Gas & Pómez Stone \\
\hline Emulsion-sol & Solid & Liquid & Cheese \\
\hline Sol-Solid & Solid & Solid & Quartz of colors \\
\hline
\end{tabular}

Scattered systems are meta-stable systems, as in balance, they tend to the total separation of phases. The stability of colloidal systems, which is identified with the necessary time to leap for phase separation to occur, depends on many factors. We can point out the composition, interfacial tension, chemical-physical nature of components, and environmental conditions, to name a few

\section{DEVELOPMENT OF METHODS CONSIDERED IN THE ANALYSIS}

\subsection{Degree of Scattering and Specific Surface Area}

The size of colloidal particles is determined by the range of attraction and repulsion forces acting between them at the microscopic level [24-26]. The formation of a system dispersed from a mixture is only possible if there are interactions between molecules to form clusters. In turn, conglomerates interact with each other to form larger particles. After a sufficiently large time, this process 
continues until total phase separation and destruction of the colloidal system occur. The rate at which this process takes place depends on the viscosity of the dispersion medium, the magnitude of the attraction between the particles, and dispersed phase concentration, which determines the distance between the particles.

Because the evolution of a colloidal system depends on particles colliding, which is a random process on the microscopic scale, particles tend to have different sizes. The size of colloidal particles is described through a probability misdrawing function $\mathrm{P}(r)$, of their average value $R$ and their standard variance or deviation. In this sense, the degree of poly dispersal is determined as: $\sigma \gamma$

$$
\gamma=\frac{\sigma}{R}
$$

where:

$\gamma<0.1$ for a mono-dispersed system, and

$\gamma>0.1$ for a poli-dispersed system.

$$
\begin{gathered}
\gamma<0.1: \text { sistema monodisperso } \\
\gamma>0.1: \text { sistema polidisperso }
\end{gathered}
$$

In addition to the degree of dispersion, another critical parameter to consider in a dispersed system is the specific surface area, defined as the total area of the interface divided by the volume or mass of the dispersed phase [27]. The specific surface area is closely related to the size of colloidal particles, and for a poly-dispersed system, with spherical particles, it is determined through the ratio [28.29]:

$$
A_{\text {esp }}=\int \frac{3}{4 R} P(r) d r\left[\frac{\mathrm{m}^{2}}{\mathrm{~m}^{3}}\right]
$$

when referred to by unit of volume, or through the equation:

$$
A_{e s p}=\int \frac{3}{4 R \rho} P(r) d r\left[\frac{\mathrm{m}^{2}}{\mathrm{~kg}}\right]
$$

For expressed by a unit of mass, where is the density of the particles. $\rho$

The specific surface area is one of the quantitative parameters used to characterize the degree of dispersion and its minimum value in the equilibrium state.

\subsection{Kinetic Properties of Scattered Systems}

Particles dispersed in a low viscosity medium are not at rest but are in constant motion. If these are small enough, the gravitational effects are negligible, and their movement is due to the random shock of these with the molecules of the dispersed phase [30]. This phenomenon is known as Brownian movement or diffusion and can eventually be appreciated on a macroscopic scale. The diffusion coefficient, which is a parameter that describes the ease of movement of particles in the medium, depends on particle size, the viscosity of the dispersion medium, and temperature. For a spherical particle, the diffusion coefficient $\mathrm{D}$ can be estimated through the ratio:

$$
D=\frac{k T}{6 \pi \mu r}
$$

where $k$ is Boltzmann's constant, $T$ is the temperature, viscosity, and $\mu r$ is the particle's radius. On a macroscopic scale, the particles move from the highest concentration zones to the areas of lower concentration, where the flow of particles per unit of measurement $J_{x}$ depends on the concentration gradient $\mathrm{C}$ and the diffusion coefficient $\mathrm{D}$ according to Fick's law:

$$
J_{x}=-D \frac{d C}{d x}
$$

If the dispersed particles are of appreciable size, they will experience the force of gravity, which manifests itself in the sedimentation process. The gravity force $F_{g}$ acting on a spherical particle depends on the difference between the densities of the medium, the solid particle, and the size of the particle:

$$
F_{g}=\frac{4}{3} \pi r^{3}\left(\rho-\rho_{0}\right) g
$$

where the particle density is and $\rho_{0}$ is the density of the dispersion medium. This force causes the particle to go down in the middle. Still, this movement is opposed to the resistance exerted by the dispersion medium. The process is associated with the viscosity of the dispersion. Viscous resistance is described through the Stokes equation:

$$
F_{\mu}=5 \pi \mu r v
$$

where $v$ is the rate of sedimentation of the particles. By matching both forces, you get an expression that allows determining the rate of sedimentation:

$$
v=\frac{4}{15} g \frac{r^{2}}{\mu}\left(\rho-\rho_{0}\right)
$$

The sedimentation rate is a property that is determined experimentally, almost always to determine the particle size [31-33]. When the sedimentation of a dispersed system occurs, a well-defined interface is seen between the already clarified dispersion medium and the region in which the particles are located, where this interface constantly decreases its height relative to time. The variable measured in this case is the variation in the size of the interface concerning time.

In dispersed mono systems, the height of interface $H$ shows linear behavior for time $t$, so that the rate of sedimentation and the size of the particles are determined through the ratio: 
L.C. Moreno-Chimely et al., International Journal of Emerging Trends in Engineering Research, 9(6), June 2021, 659 - 664

$$
\begin{gathered}
v=\frac{H_{t=0}-H_{t=t_{r}}}{t_{r}} \\
r=1.9365 \sqrt{\frac{v \mu}{g\left(\rho-\rho_{0}\right)}}
\end{gathered}
$$

If the solution is poly disperses, the height of the interface does not show linear behavior, and the determination of the sedimentation rate is more complicated and is based on the following procedure:

1. Observe the height behavior of the interface relative to time and adjust the observed data to a statistical model $H(t)$ of linear regression of order greater than 1.

2. Determine the fraction for particles of larger dimensions than settled for a t-time from the adjusted model:

$$
q(t)=H(t)-t \frac{d H(t)}{d t}
$$

3. The particle size distribution curve, which is expressed as a function of time, is determined as:

$$
\begin{aligned}
x_{q}(t) & =-\frac{d q(t)}{d t} \\
& \equiv \frac{\text { partículas de radio } r_{x}}{\text { partículas totales }}
\end{aligned}
$$

4. For a selected $x_{q}$, the corresponding time value is cleared. With the time value, the curve $H(t)$ is set to determine the height of the corresponding interface so that the sedimentation rate and particle size are defined as:

$$
\begin{gathered}
v_{x}=\frac{H\left(t=t_{x_{q}}\right)}{t_{x_{q}}} \\
r_{x}=\sqrt{\frac{9 \mu}{2 g\left(\rho_{s}-\rho_{l}\right)} v_{x}}
\end{gathered}
$$

5. Repeat step 4 until the velocity distribution curve, and particle sizes are found.

These kinetic properties do not manifest when the dispersion medium is a solid or liquid of a very high viscosity so that the displacement of particles can be considered negligible.

\subsection{The viscosity of Suspensions and Emulsions}

It has been theoretically and experimentally demonstrated that the viscosity and density of a dispersed system depend on the volume fraction of the dispersed phase. The thickness of the dispersed system is always greater than the viscosity of the dispersion medium, while the density depends on the density of the phases involved and the composition of the system and maybe greater or lower than the density of the dispersion medium [34]. For suspensions, density and viscosity are determined through the ratios:

$$
\begin{gathered}
\rho=\rho_{l}+\left(\rho_{s}-\rho_{l}\right) v_{s} \\
\mu=\mu_{l}\left(1+2.5 v_{s}\right): v_{s} \leq 0.05 \\
\mu=\mu_{l} \exp \left(\frac{\frac{5}{2} v_{s}}{1-\frac{v_{s}}{0.53}}\right): v_{s}>0.05
\end{gathered}
$$

where while in the case of emulsions, these will be given by:

$$
\begin{gathered}
\rho=\rho_{m}\left(1-v_{d}\right)+\rho_{d} v_{d} \\
\mu_{e}=\mu_{m}\left(1+\left(\frac{\mu_{m}+\frac{5}{2} \mu_{d}}{\mu_{m}+\mu_{d}}\right) v_{d}\right)
\end{gathered}
$$

\section{STUDYING PROPERTIES AND OBTAINING NEW BUILDING MATERIALS BY THE THEORY OF DISPERSED SYSTEMS}

When researching obtaining new building material from certain raw materials, it is necessary to carry out an experimental study that relates the composition of the material with physical properties such as compressor resistance, the elasticity module, and thermal conductivity. Because these properties are related to porosity, adding a binder product causes attraction between particles to increase so that final porosity decreases.

In this sense, to study the effect of the binder, sedimentation tests should be carried out, which allow knowing the particle size distribution and viscosity analysis, which are also related to the volume fraction of suspended solids. For the material study, it is necessary to form a mixture with an excess of water. If you work with the mix that will subsequently be dried to obtain the material, no sedimentation should be observed.

Another analysis that will be interesting to perform is studying the porosity associated with open pores, which can be seen employing images of the surface taken on a microscopic scale.

It is then determined whether there is a correlation between these measurements and the properties of materials, such as compressive resistance and elasticity, to understand better the chemical-physical processes that occur and improve the properties of the final product.

In this way, a vast number of considerations have been proposed in this paper to analyze these materials.

\section{CONCLUSION}

This article chooses a set of equations needed in the materials characterization considering a dispersed system perspective. 
L.C. Moreno-Chimely et al., International Journal of Emerging Trends in Engineering Research, 9(6), June 2021, 659 - 664

The behavior is related to most of the mixtures used in the construction industry and materials science. In many cases, fluidity mixtures are first obtained and, during the solidification process, behavior determines the final properties of them, such as mechanical resistance and hardness. We also present steps that can be followed in this evaluation for implementation at the various levels of study.

\section{REFERENCES}

1.Ríos-Parada, V., Jiménez-Quero, V. G., Valdez-Tamez, P. L., \& Montes-García, P. (2017). Characterization and use of an untreated Mexican sugarcane bagasse ash as supplementary material for the preparation of ternary concretes. Construction and Building Materials, 157, 83-95.

2.Fediuk, R. S., Yevdokimova, Y. G., Smoliakov, A. K., Stoyushko, N. Y., \& Lesovik, V. S. (2017, July). Use of geonics scientific positions for designing of building composites for protective (fortification) structures. In IOP Conference Series: Materials Science and Engineering (Vol. 221, No. 1, p. 012011). IOP Publishing.

3.Watrobska-Swietlikowska, D. (2020). Distribution of benzalkonium chloride into the aqueous phases of submicron dispersed systems: emulsions, aqueous lecithin dispersion and nanospheres. Aaps Pharmscitech, 21(1), 1-10.

4.MacLeod, A. J., Fehervari, A., Gates, W. P., Garcez, E. O., Aldridge, L. P., \& Collins, F. (2020). Enhancing fresh properties and strength of concrete with a pre-dispersed carbon nanotube liquid admixture. Construction and Building Materials, 247, 118524.

5.Murciego Murciego, A. (2016). Manual básico de reconocimiento macroscópico de algunos minerales y rocas.

6.Darias, J. R. (2014). Los materiales granulares: una mirada al interior del pimentero. Acta científica venezolana, 62-72.

7.Ordenes, R., Arellano, M., Jara, R., \& Merino, C. (2014). Representaciones macroscópicas, submicroscópicas y simbólicas sobre la materia. Educación química, 25(1), 46-55.

8.Mikhailov, E. F., Merkulov, V. V., Vlasenko, S. S., Ryshkevich, T. I., \& Pöschl, U. J. (2011). Filter-based differential hygroscopicity analyzer of aerosol particles. Izvestiya, Atmospheric and Oceanic Physics, 47(6), 747-759.

9.Kislyj, P. S., Storozh, B. D., \& Gorb, M. L. (1977). Strength of aluminium oxide-tungsten cermets. Poroshk. Metall, 75-79.

10. Koryanova, Y. I. (2017). Structure and properties of concrete for injection with two-stage expansion. In Solid State Phenomena (Vol. 265, pp. 103-108). Trans Tech Publications Ltd.

11. Mott, R. L., Salas, R. N., Flores, M. A. R., \& Martínez, E. B. (2009). Resistencia de materiales (Vol. 5). Pearson Educación.

12. Boccaccini, A. R., Ondracek, G., Mazilu, P., \& Windelberg, D. (1993). On the effective Young's modulus of elasticity for porous materials: microstructure modelling and comparison between calculated and experimental values. Journal of the Mechanical Behavior of Materials, 4(2), 119-128.
13. Phani, K. K., \& Niyogi, S. K. (1987). Young's modulus of porous brittle solids. Journal of materials science, 22(1), 257-263.

14. Chuc, N. T., Van, L. T., \& Bulgakov, B. I. (2018). Designing the composition of concrete with mineral additives and assessment of the possibility of cracking in cement-concrete pavement. In Materials Science Forum (Vol. 931, pp. 667-673). Trans Tech Publications Ltd.

15. Gallegos-Villela, R. R., Larrea-Zambrano, F. D., Goyes-Lopez, C. E., Perez-Sanchez, J. F., Suarez-Dominguez, E. J., \& Palacio-Perez, A. (2021). Effect of natural additives on concrete mechanical properties. Cogent Engineering, 8(1), 1870790.

16. Roussel, N. (Ed.). (2011). Understanding the rheology of concrete. Elsevier.

17. Zhang, H. L., Tang, Y., Meng, T., \& Zhan, L. T. (2021). Evaluating the crushing characteristics of recycled construction and demolition waste for use in road bases. Transportation Geotechnics, 28, 100543.

18. Ye, H., Cai, R., \& Tian, Z. (2020). Natural carbonation-induced phase and molecular evolution of alkali-activated slag: Effect of activator composition and curing temperature. Construction and Building Materials, $248,118726$.

19. Xu, G., \& Shi, X. (2018). Characteristics and applications of fly ash as a sustainable construction material: A state-of-the-art review. Resources, Conservation and Recycling, 136, 95-109.

20. Slomkowski, S., Alemán, J. V., Gilbert, R. G., Hess, M., Horie, K., Jones, R. G., ... \& Stepto, R. F. (2011). Terminology of polymers and polymerization processes in dispersed systems (IUPAC Recommendations 2011). Pure and Applied Chemistry, 83(12), 2229-2259.

21. Spasic, A. M., \& Lazarevic, M. P. (2007). A new approach to the phenomena at the interfaces of finely dispersed systems. Journal of colloid and interface science, 316(2), 984-995.

22. Matveenko, V. N., \& Kirsanov, E. A. (2011). The viscosity and structure of dispersed systems. Moscow University Chemistry Bulletin, 66(4), 199-228.

23. Spasic, A. M. (2018). Classification and Methodology_Finely Dispersed Systems. In Interface Science and Technology (Vol. 22, pp. 41-49). Elsevier.

24. Bowen, W. R., \& Jenner, F. (1995). The calculation of dispersion forces for engineering applications. Advances in colloid and interface science, 56, 201-243.

25. Dickinson, E. (2003). Hydrocolloids at interfaces and the influence on the properties of dispersed systems. Food hydrocolloids, 17(1), 25-39.

26. Lin, S., Shih, C. J., Sresht, V., Rajan, A. G., Strano, M. S., \& Blankschtein, D. (2017). Understanding the colloidal dispersion stability of 1D and 2D materials: perspectives from molecular simulations and theoretical modeling. Advances in colloid and interface science, 244, 36-53.

27. Uriev, N. B. (2016). Technology of Dispersed Systems and Materials. John Wiley and Sons, Incorporated. 
28. Sears, G. W. (1956). Determination of specific surface area of colloidal silica by titration with sodium hydroxide. Analytical Chemistry, 28(12), 1981-1983.

29. Yukselen, Y., \& Kaya, A. (2006). Comparison of methods for determining specific surface area of soils. Journal of Geotechnical and Geoenvironmental Engineering, 132(7), 931-936.

30. Matveenko, V. N., \& Kirsanov, E. A. (2011). The viscosity and structure of dispersed systems. Moscow University Chemistry Bulletin, 66(4), 199-228.

31. Huang, Y., Li, F., Liu, Z., Li, J., \& Gao, X. (2021). Experimental determination of sediment transport capacity of concentrated water flow over saturated soil slope. European Journal of Soil Science, 72(2), 756-768.

32. Adeniji, A. O., Okoh, O. O., \& Okoh, A. I. (2017). Analytical methods for the determination of the distribution of total petroleum hydrocarbons in the water and sediment of aquatic systems: A review. Journal of Chemistry, 2017.

33. Yoobanpot, N., Jamsawang, P., Simarat, P., Jongpradist, P., \& Likitlersuang, S. (2020). Sustainable reuse of dredged sediments as pavement materials by cement and fly ash stabilization. Journal of Soils and Sediments, 20, 3807-3823.

34. Liu, Y., \& Xi, Y. (2019). Colloidal systems with a short-range attraction and long-range repulsion: Phase diagrams, structures, and dynamics. Current Opinion in Colloid \& Interface Science, 39, 123-136. 